\title{
Mirroring the voice from Garcia to the present day: Some insights into singing voice registers
}

\author{
NATHALIE HENRICH \\ Laboratoire d'Acoustique Musicale, Paris, France
}

\begin{abstract}
Starting from Garcia's definition, the historical evolution of the notion of vocal registers from then until now is considered. Even though much research has been carried out on vocal registers since then, the notion of registers is still confused in the singing voice community, and defined in many different ways. While some authors consider a vocal register as a totally laryngeal event, others define it in terms of overall voice quality similarities. This confusion is reflected in the multiplicity of labellings, and it lies in the difficulty of identifying and specifying the mechanisms distinguished by these terms. The concept of laryngeal mechanism is then introduced, on the basis of laryngeal transition phenomena detected by means of electroglottography. It helps to specify at least the laryngeal nature of a given singing voice register. On this basis, the main physiological, acoustic, and perceptual characteristics of the most common singing voice registers are surveyed.
\end{abstract}

Key words: Laryngeal mechanism, singing voice, vocal register

\section{Introduction}

Before the nineteenth century, knowledge about human phonation was limited to what could be perceived by auditory perception and proprioceptive sensations, or observed from outside during phonation and inside on cadavers. The singing voice registers are a known fact, related to perception of different voice qualities. In this context, the singing voice teacher Manuel Garcia II (1805-1906) shows not only an interest in the voice sound quality but also in the underlying vocal physiology mechanisms. On November 16th, 1840, he presented the results of his observations as a voice teacher and his experiments on the human voice to the French Académie des Sciences (1), prior to the publication of his famous singing voice method Ecole de Garcia: Traité complet de l'art du chant (2). In this paper, he claims that the human voice is composed of different registers: poitrine (chest), fausset-tête (falsetto-head), and contre-basse (counter bass). He defines the registers as follows: 'By the word register we mean a series of consecutive and homogeneous tones going from low to high, produced by the same mechanical principle, and whose nature differs essentially from another series of tones equally consecutive and homogeneous produced by another mechanical principle. All the tones belonging to the same register are consequently of the same nature, whatever may be the modifications of timbre or of the force to which one subjects them'. ${ }^{1}$ Therefore, Garcia pioneers the definition of vocal registers in relation to a given mechanical principle, independently of any timbre variation. In his definition of vocal registers, the concept of a mechanical principle prevails over the perceptual dimension. Basing his rationale on physiological observations of the larynx position, he demonstrated that the falsetto and head registers result from the same laryngeal mechanism, their difference being a timbre effect. ${ }^{2}$

Nowadays, we have experimental techniques to which Garcia did not have access at that time. How has the knowledge about singing voice registers evolved since Garcia's time? How are the vocal registers defined and identified? What are their physiological, acoustic and perceptual attributes? By reviewing the historical evolution of the notion of vocal register, this paper aims to provide a possible

Correspondence: Dr. Nathalie Henrich, Institut de la Communication Parlée, 46 avenue Félix Viallet, 38031 Grenoble Cedex 01, France. E-mail: henrich@icp.inpg.fr 


\section{N. Henrich}

guide for a better understanding of this notion. A brief overview of the evolution of definition, numbers and labellings will be given. The different means of identifying a vocal register will be discussed, and the concept of laryngeal mechanism will be introduced, on the basis of laryngeal transition phenomena. Finally, the most common singing voice registers will be reviewed, and their physiological, acoustic and perceptual properties will be given.

\section{Historical evolution of the notion of vocal register since Garcia}

From Garcia to the present time, the notion of vocal registers has evolved in many ways. In particular, this evolution concerns the definition of a vocal register, the number of registers identified and their labelling.

\section{From Garcia to the 1960s}

As mentioned in the introduction, the singing teacher Manuel Garcia in 1847 is considering three main different registers, chest, falsetto-head and contre-basse, the other possible registers, such as voix mixte, sharing similar mechanical principle with these main ones (for a detailed discussion about Garcia's falsetto-head register, we refer the reader to (3)). The frequency ranges of these registers are shown in Figure 1. Interested in understanding the physiology of these registers, Garcia explores his own larynx and those of a few of his students by using a laryngoscope, a clever observation tool which would give rise to our present endoscopic camera. It consists of a small mirror fixed to the end of a long handle and placed at the back of the throat (see Figure 2). When the throat is lighted in the proper way, the glottis may be observed from above. The laryngoscope was already available in the early nineteenth century-Babington used it in 1827 (4) - so Garcia did not invent it, but he was the first to use it successfully, being able not only to see the vocal folds at rest, but also in the act of singing. Garcia's observations were communicated to the Royal Society by Dr Sharpey on May 24th, 1855 $(4,5)$.

In 1880, the physiologist and voice production teacher Emil Behnke and the throat surgeon Lennox Browne also made use of the laryngoscope to obtain in vivo images of the glottis. Behnke defines the vocal registers as follows: 'a register consists of a series of tones which are produced by the same mechanism. [...] There are, broadly speaking, three registers in the human voice, and the mechanisms are plainly visible, as follows: 1) During the lowest series of tones the vocal ligaments vibrate in their entire thickness. 2) During the next series of tones the vocal ligaments vibrate only with their thin inner edges. 3) During the highest series of tones a portion of the vocal chink is firmly closed, and only a small part of the vocal ligaments vibrates.' ((4), p. 86). In this definition, similarly to Garcia's, the concept of laryngeal mechanisms is underlying. On the basis of these physiological observations, he adopts Curwen's thick, thin and small labelling, and he describes three registers for the male voice (lower thick, upper thick and upper thin) and five registers for the female voice

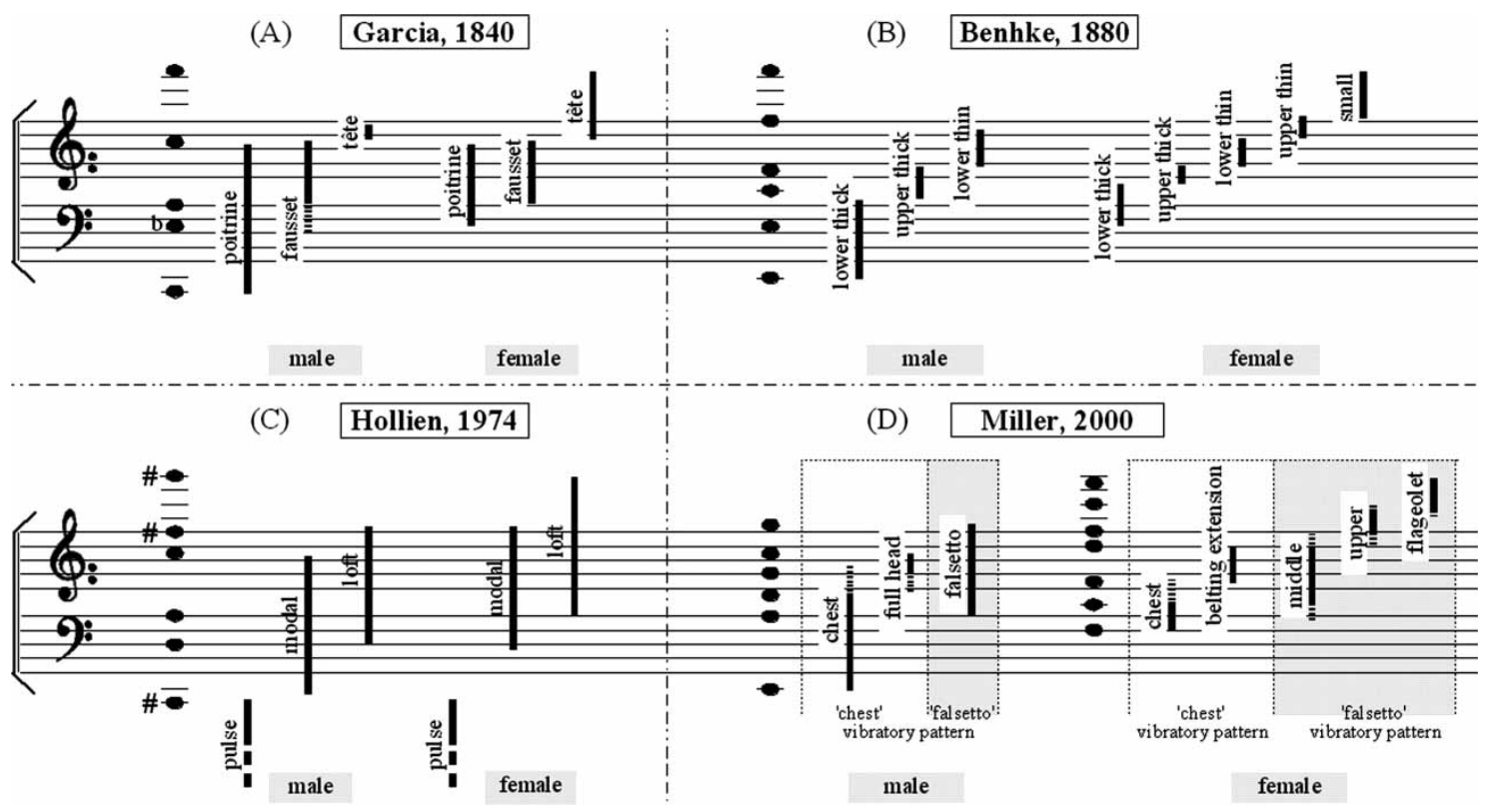

Figure 1. Frequency range of human voice and vocal registers, as defined by different authors: (A) Garcia (1), (B) Behnke (4), (C) Hollien (11), (D) Miller (17). 


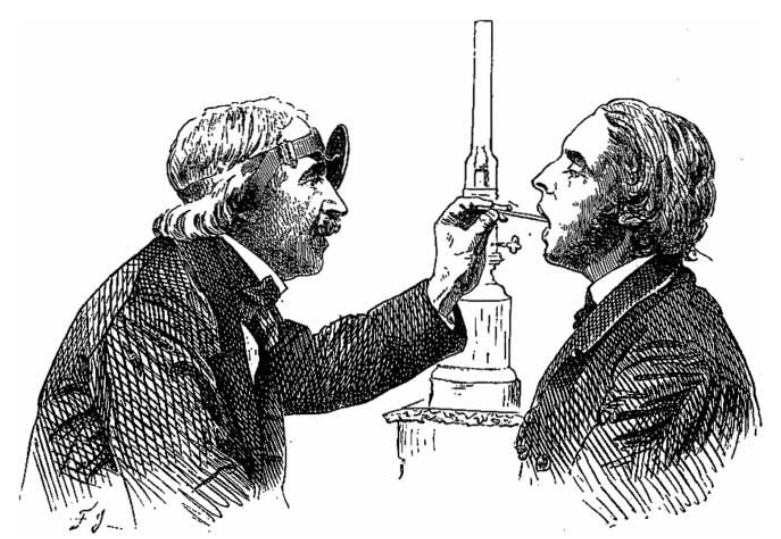

Figure 2. The laryngoscope. From (2).

(lower thick, upper thick, lower thin, upper thin and small). Their corresponding frequency ranges are shown in Figure 1. The transition between thick and thin registers is characterized by a break which occurs around F4 or F4\# in both male and female voices, individual differences apart.

In the early twentieth century, whereas few singing teachers maintain that a 'natural' voice has only one single register $(6,7)$, the general tendency is to accept the existence of at least two and at maximum five registers. The term 'register' is even sometimes replaced by 'mechanism', for example by Wilcox $(6,7)$ who suggests the terms 'heavy mechanism' and 'light mechanism'.

The terms used to label the vocal registers are abundant and author dependent, and most of the time their usage is ambiguous. In 1963, a literature survey summary concerning the pitch range and labelling of voice registers (8) mentions that 'the only secure common denominator for defining a register is by means of its range on the musical scale.' Reasonable agreement is found on 'the average pitch of the boundaries between registers, i.e. the breaks or voice transitions.' It is also mentioned that these average boundaries do not vary much with the type of voice, and that the nature of the breaks is 'still a matter of controversy'.

The two most common registers are labelled as chest register and head or falsetto register. The distinction between head and falsetto is very much author dependent. In his time, Garcia placed the falsetto register between chest and head register, and it may thus correspond to a middle register, as suggested by Vennard (7). Yet, when the head register is recognized as a distinct register, it is very often placed between chest and falsetto. Otherwise, either it is put aside, or it replaces the falsetto register, in the case of female voice for instance. The two main registers overlap in a frequency region where a third register is sometimes mentioned, and
Mirroring the voice from Garcia to the present day

labelled as medium, mid, middle, or mixed register. It may correspond to the French voix mixte or the Italian voce mista. Two additional registers are mentioned at the extreme low and high ends of the frequency range: the Strohbass register, and the bell, flute or whistle register.

\section{From the 1960s to the 1980 s}

From the early 1960 s to the 1980 s, a good deal of research effort was dedicated to the understanding of the acoustic, mechanical, and physiological properties of these registers. Working on excised larynges, the physicist Janwillem Van den Berg 1963 explored the vibratory pattern of the vocal folds and the influence of the subglottal resonance. He came to the conclusion that the mid register may not be considered as a distinct register (9). The physician Minoru Hirano, together with two voice teachers William Vennard and John Ohala, investigated the role of intrinsic laryngeal muscles using electromyography (10). They found that the vocalis muscle is essential to register regulation, its activity being greater in heavy register. In particular, the vocalis muscular activity decreases during a register shift from heavy to light and increases from light to heavy. The lateral cricoarytenoid, interarytenoid and cricothyroid muscles also contribute to register regulation, but to a smaller extent.

In 1974, the speech scientist Harry Hollien reconsiders the definition, numbering and terminology of registers (11). He defines a vocal register 'as a series or range of consecutively phonated frequencies which can be produced with nearly identical vocal quality and that ordinarily there should be little or no overlap in fundamental frequency between adjacent registers. Furthermore, [he maintains] that a voice register is a totally laryngeal event and, before the existence of a particular register can be established, it must be operationally defined: 1) perceptually, 2) acoustically, 3) physiologically and 4) aerodynamically.' With regard to this definition based on a laryngeal entity, he considers three major registers, which have been experimentally validated, and to which he gives unfamiliar but also 'uncontaminated' labels: pulse, modal and loft registers. The pulse register includes vocal fry, creak and strohbass registers; the modal register includes chest, head, low, mid and high; the loft register is the equivalent of the phoneticians' and speech pathologists' falsetto register. Their corresponding frequency ranges are shown in Figure 1. Hollien mentions a fourth register, the flute, whistle or pipe, which he puts aside because of a lack of empirical information and experimental exploration. 


\section{N. Henrich}

Hollien's significant paper has pointed out the confusion around the concept of vocal registers among the voice community, and it may have contributed to the emergence of an international discussion. In the late $1970 \mathrm{~s}$, spurred on by the Collegium Medicorum Theatri (CoMeT), an international organization composed of physicians, voice scientists, voice coaches and voice pathologists, a committee on vocal registers was formed in an attempt to clarify the notion of vocal registers and to find a consensual position among the international voice community. The committee agreed on five points, which are reported by Hollien (12):

1. Registers exist: voice registers exist in the speaking and singing voice, and they 'must be recognized as an entity'. Their perceived acoustic effects may be compensated for by appropriate training, if this 'is considered desirable'.

2. Singing/speaking registers: 'vocal registers in singing and voice registers in speaking (or in the untrained voice) are different and separate entities and must be treated as such'. However, they 'may overlap in function and probably have common physiological roots in the larynx'.

3. To 'remove' registers: two postulates are stated by the committee. On the one hand, 'eliminating or concealing register effects is desirable for the classical/western, concert/opera mode of singing'. On the other hand, 'register effects can be fundamental to certain types of singing'. It is also important to keep in mind that 'voice registers cannot be removed as they are physiologically given.'

4. The source of registers: First, the committee has 'accepted the notion that there probably are two sources for registers - the larynx and the vocal tract'. However, this point seems to have raised a great debate among the committee members. 'A substantial minority of the committee argued in favor of the source (of a voice register) being only laryngeal and that the other so-called register-like phenomena actually are some sort of quality/timbre events.'

This latter position is in line with Garcia's definition. Unfortunately, no mention of what defines and identifies a register in the human voice is given in the report. This may explain the controversy about the question of the source of registers. As a consequence, a great debate was also raised by the labelling question.

5. Labels: The committee has rejected the use of so called 'old terms', and in particular chest and head which are based upon singers' sensations. Two main proposals are made. The first suggestion is to number them: '\#1: for the very lowest of registers, probably used only in speaking (old terms: pulse, vocal fry, creak), \#2: that (low) register, which is used for most speaking and singing (old terms: modal, chest, normal, heavy), \#3: a high register used primarily in singing (old terms: falsetto, light, head), \#4: a very high register usually found only in some women and children and not particularly relevant to singing (old terms: flute, whistle).' An additional register is referred to as ' $\# 2 \mathrm{~A}^{\mathrm{\prime}}$ ' and defined as "that "register" which is described by many voice teachers as in the middle of the frequency range [...] (old terms: head, mid, middle, upper).' The second suggestion is to favour 'new terms, generic terms, those that are clear and easy to understand', such as the pair of terms heavy, light, or lower, upper.

\section{From the 1980s to the present time}

Despite this great effort at clarification, the voice community remains divided on the question of vocal registers. The existence of at least two main laryngeal vibratory mechanisms has been experimentally validated. Among the speech community, an implicit agreement is found on three registers (pulse or vocal fry, modal or chest, and falsetto registers), in relation to specific laryngeal adjustments. In the singing voice community, the definition, numbers and labelling of registers are still a matter of debate, and they continue to vary among authors. Indeed, vocal registers have an acoustical and perceptual reality for singers, which cannot be ignored. On the one hand, a vocal register is defined by its laryngeal mechanical properties (13-15), following in this sense Garcia, Benhke and Hollien. As an example, Sakakibara (15) proposes the following definition: 'the vocal register is a set or range of serial sounds that are similar in perception and produced by similar vocal fold vibratory patterns.' On the other hand, a vocal register is defined by its characteristic voice quality, following in this sense Large ((16), cited in (17)). As an example, Titze mentions that "the term "register" has been used to describe perceptually distinct regions of vocal quality that can be maintained over some ranges of pitch and loudness.'((18), p. 253) These two approaches of the notion of vocal register are sometimes implicitly combined. In a recent thesis on registers in singing (17), the singer and voice teacher Donald Miller points out the distinction between registration defined 'as an exclusive feature of the voice source' and the 'integrated approach to registration, including adjustments of the vocal tract, as well as those of the source'. He chooses the latter approach, but defines the 'natural registers' as registers related mainly to 
glottal source adjustments. These natural registers are designated 'chest' and 'falsetto', and the author refers to the 'chest vibratory pattern' and 'falsetto vibratory pattern' when emphasizing the voice source characteristics of a given register. According to this author, the female voice is divided into five registers: chest and belting registers, which both have the chest vibratory pattern, and middle, upper and flageolet registers, which all have the falsetto vibratory pattern. The male voice is divided into four registers: chest, full head and mezza voce registers, which all have the chest vibratory pattern, and falsetto register, which has the falsetto vibratory pattern. The corresponding frequency ranges are shown in Figure 1.

\section{Conclusion: what are the vocal registers?}

In his time, Garcia was distinguishing vocal registers on the basis of a 'mechanical principle' which he related to a laryngeal event. Following Garcia's path, other authors, such as Benhke, Hollien or Roubeau, have explored the concept of laryngeal-related vocal registers with the help of more and more sophisticated experimental techniques: laryngoscopy, cinematography, electromyography, electroglottography (EGG), etc. The understanding of laryngeal vibratory mechanisms has become more refined.

Yet, a vocal register classification based only on laryngeal phenomena does not take into account the great timbre variations which can be achieved in singing. This may explain that, even today, the notion of vocal registers in singing remains very often a matter of debate among the singing voice community. Whereas some authors define the registers exclusively on the basis of an underlying laryngeal mechanical principle, others prefer to define them on the basis of sound voice quality. In this context, to find a consensual position on the labelling of registers is inevitably problematic, but the core of this problem is less the terminology itself than the 'failure to identify and specify the mechanisms distinguished by the terms', as pointed out by Miller ((17), p. 32).

In the next section, we will see how the problem of identifying the mechanisms related to singing voice registers has been partly solved, at least at the laryngeal level.

\section{Transition phenomena: how to identify a laryngeal mechanism}

The reason for defining at least two registers in human phonation comes from the discontinuities or transition phenomena, which can occur voluntarily or involuntarily during the production of voiced sounds. Different kinds of transition phenomena are listed in the literature, in particular periodicity, timbre, and laryngeal transitions. They will be briefly presented here. We will then focus on the laryngeal vibratory mechanisms, the consistency of which is evinced by laryngeal transition phenomena.

\section{Detection of transition phenomena}

A transition phenomenon may result from psychoacoustic properties of human perception. The 'periodicity transition' distinguished by Titze $(18,19)$ is an example of such a transition. It refers to 'changes in vocal quality that occur whenever glottal pulses are perceived as individual events rather than a continuous auditory stimulus' (19). According to this author, it accounts for the distinction between pulse (or vocal fry) and chest registers. A given voice production is perceived as pulsed when its fundamental frequency $F_{0}$ is lower than a $70-\mathrm{Hz}$ 'crossover' frequency, and perceived as non-pulsed above this frequency. In the case of a $F_{0} / n$ subharmonic pattern, the crossover frequency is $\mathrm{n} \times 70 \mathrm{~Hz}$. According to Titze, the reason for this perceptual fact lies in formant energy damping (related to the formant bandwidth) over the fundamental period: waveforms are perceived as pulse-like if the formant energy during one excitation is sufficiently damped out before the next excitation occurs. This author defines the pulse-chest registers boundaries on the basis of this periodicity transition.

Transition phenomena may also result from noticeable timbre variations $(17,19,20)$, which may or may not be related to a laryngeal adjustment.

According to Titze $(18,19)$, timbre transitions are characterized by an abrupt voice quality change, which is associated with a spectral energy change in the high-frequency part of the sound spectrum, i.e., a modification in the spectral slope. This spectral change may come from a variation in the glottal flow derivative discontinuity and the return phase, which can be more or less abrupt. From the acoustic point of view, it may result from an interference between a subglottal resonance and the vocal fold driving pressure.

According to Miller (17), either these transitions have a laryngeal source, or they result from resonance strategies, also called 'formant tuning'. In this latter case, a vocal tract resonance is adjusted to match a given harmonic in frequency, reinforcing the energy in this harmonic frequency band. The tuning process may induce the presence of strong acoustic standing waves, which in return can affect the vocal fold vibratory movement, yet in a more subtle way than in the case of a laryngeal adjustment (17). 
It is difficult to give strong evidence of these vocal tract adjustments, as the vocal tract resonances are difficult to measure reliably from the acoustic signal, especially when the harmonic spacing is broad. On the contrary, the laryngeal transition phenomena, which are related to a sudden adjustment of the glottal vibratory pattern, are more easily detectable. For instance, the pitch jump is the most common and obvious transition phenomenon which can occur during male and female phonation, in speech and singing (13,21-23). Avoided in the classical singing technique, it is used as an ornament or even a singing style in other vocal cultures, such as the Tyrolean or African yodel $(20,24)$. Evidence has been given that this transition phenomenon is only related to a biomechanical laryngeal adjustment, as it can occur on excised larynges without the presence of vocal tract loading (25). As illustrated in Figure 3, a vocal fold is a heterogeneous layered structure (26), composed of the cover (mucosa, epithelium and superficial layer of lamina propria), the transition (intermediate and deep layers of lamina propria, also called vocal ligaments), and the body (vocalis muscle). The abruptness of the frequency jump is accounted for by a sudden variation of the vibrating mass in action, induced by a decoupling of the vibrating layers due to a modification in vocal fold tension (13). As this modification in tension may be gradual, these frequency jumps are considered to be manifestations of bifurcations in the vocal fold vibratory mechanism (23).

A noticeable frequency jump often goes with a jump in the open quotient $(21,27)$, which can be detected on electroglottographic signals. This jump occurs, for instance, during the production of a glissando, as illustrated in Figure 4. The open

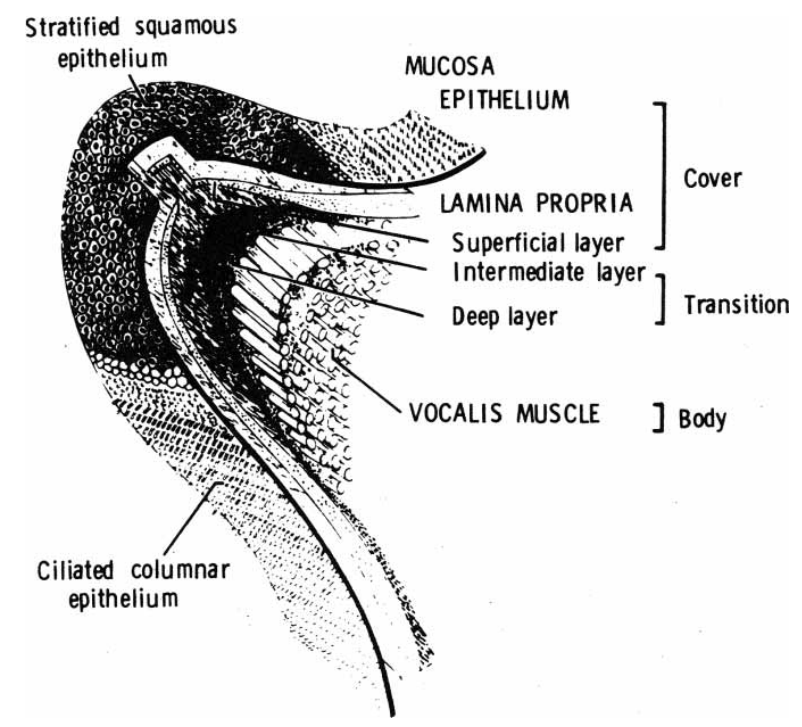

Figure 3. The vocal-fold layered structure. From (26). quotient jump is one characteristic of a laryngeal transition. As illustrated in Figure 4, it may occur even in the case when the singer manages to smooth the transition, thus avoiding the frequency jump (27).

Laryngeal transition phenomena can be characterized by a noticeable change in the vocal fold contact area, which can easily be detected using electroglottography by an abrupt EGG amplitude variation $(22,28)$. On the basis of these transition phenomena detected by electroglottography, the concept of laryngeal mechanism has been defined $(13,22,29)$. This concept, which is of great help in clarifying the notion of vocal registers in terms of laryngeal configuration and glottal vibratory properties, will now be summarized.

\section{The laryngeal mechanisms}

The variation in EGG amplitude which goes with a noticeable register break $(28,30)$ can similarly be observed during a register transition without any noticeable break $(22,29)$. Therefore, a register transition associated with a laryngeal adjustment can easily be identified by an amplitude change in the EGG signal, as illustrated in Figure 4. Even when the singer is skilled enough to smooth the transition, so that no break or timbre change can be noticed, the vocal register transition can be detected on the EGG signal.

On the basis of these laryngeal-transition phenomena, the notion of laryngeal mechanism has been defined $(13,22,29)$. The choice has been made to number them, following in this way one of the 1983 CoMeT labelling positions (12). During the production of a glissando from the lowest to the highest possible pitches, three laryngeal transitions can be detected, as shown in Figure 5. These transitions delimit four frequency regions, in which the voice is produced by the use of a precise laryngeal vibratory mechanism (laryngeal mechanism M0 to M3).

A laryngeal mechanism can be considered as a substitute for a vocal register which would totally be defined as a laryngeal entity. The corresponding register counterparts of the laryngeal mechanisms are given in Table I.

The laryngeal mechanisms correspond to different vocal fold configurations which will now be described. A schematic representation of the two main ones, M1 and M2, is plotted in Figure 6.

In laryngeal mechanism M0, the vocal folds are very short and thick, all the layers being slackened and pliant $(11,26)$. Vocalis, cricothyroid, and interarytenoid muscular activities are minimal, as compared to other laryngeal mechanisms $(26,31)$. The closed phase is usually very long. The lowest 

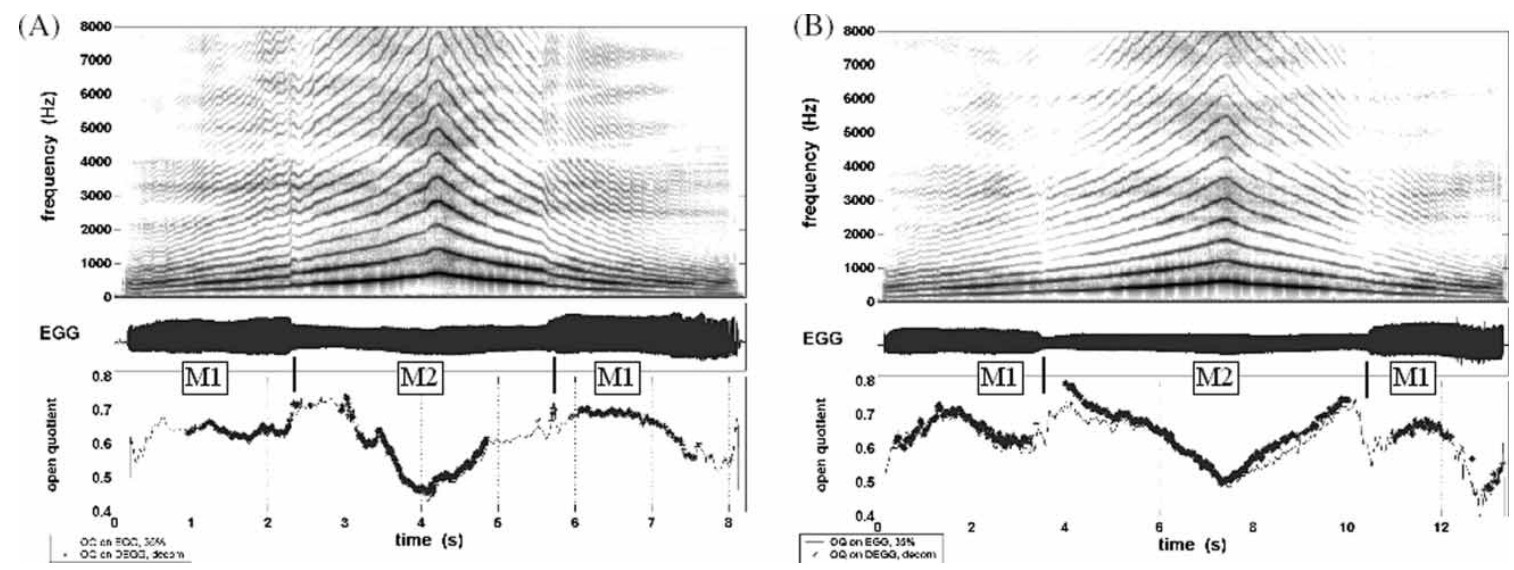

Figure 4. Illustration of vocal registers transition phenomena on a glissando sung by a counter tenor (A) with a pitch jump and a noticeable timbre change, (B) without any noticeable break. The top panel shows the time-frequency analysis, the middle panel the EGG signal, and the bottom panel the EGG measured open quotient. Two methods have been used to measure open quotient: on the EGG signal, using a $35 \%$ threshold method, and on the DEGG signal, using the DECOM method (55). The determination of the underlying laryngeal mechanism (M1 or M2) is based on the EGG detected laryngeal transitions.

frequencies are usually produced in this laryngeal mechanism.

In laryngeal mechanism M1, the vocal folds are thick and they vibrate over their whole length with a vertical phase difference (7). Vibrating mass and amplitude are important. The vocal fold body is stiffer than the cover and transition (26). Vocalis muscular activity is dominant over cricothyroid $(26,31)$. Both activities increase with pitch. Closed phase is often longer than open phase, which is reflected in the open or closed quotient values (27). This laryngeal mechanism is used by both males and females in the low to mid part of their frequency range. The corresponding average voice range profiles for males and females are shown in Figure 7.

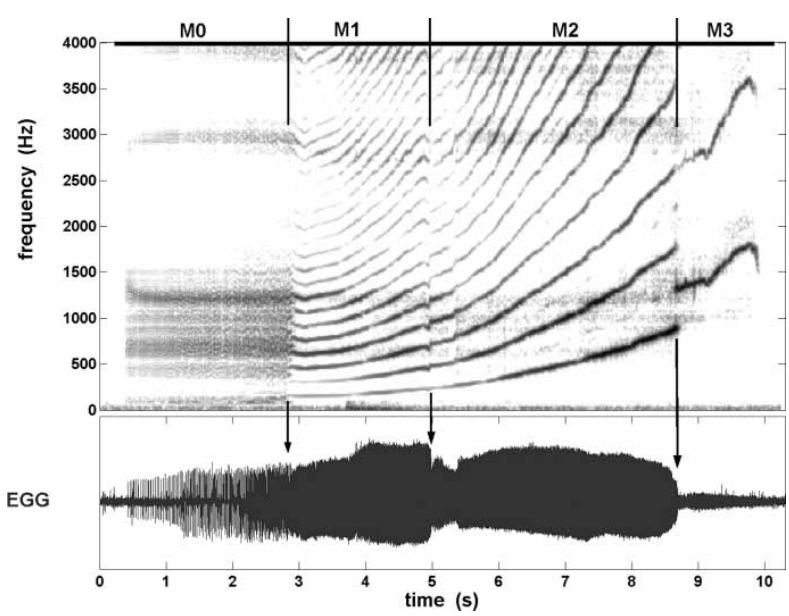

Figure 5. Illustration of the four laryngeal vibratory mechanisms on the production of a glissando. The top panel shows the time frequency analysis, and the bottom one the EGG signal.
In laryngeal mechanism M2, the vibrating mass and amplitude are reduced (7), as compared to mechanism $M 1$, and there is no vertical phase difference in the glottal vibratory movement. All the vocal fold layers are stretched, and the collagenous fibres in the vocal ligament are the stiffest of all the layers (26). Cricothyroid muscular activity is dominant over vocalis. The open phase is always longer than the closed phase, lasting at least $50 \%$ of the fundamental period (27). This laryngeal mechanism is used by both males and females in the mid to high part of their frequency range. The corresponding average voice range profiles for males and females are shown in Figure 7.

The laryngeal mechanism M3 is far from being understood. Whistle, flute or flageolet registers may be produced in mechanism M3, and only few studies have been dedicated to these registers. In this mechanism, the vocal folds are thin and very tensed. The vibratory amplitude is much reduced as compared to mechanism M2, and there even may be no contact in some cases. It has been suggested that the vocal fold vibration may be induced by periodic vortex shedding interacting with the resonator (32).

The laryngeal mechanisms are not related exclusively to speech or singing, but they are a physiological entity in human phonation (22). They are also common to male and female, who share similar laryngeal mechanism boundaries (see Figure 7). A characteristic of the human voice is that the frequency regions of the main mechanisms M1 and M2 can overlap over a precise frequency range (33), as shown in Figure 7: E3 $(165 \mathrm{~Hz})$ to $\mathrm{F} \# 4(370 \mathrm{~Hz})$ for male voices, and G3 $(196 \mathrm{~Hz})$ to $\mathrm{G} 4(392 \mathrm{~Hz})$ for female voices. At those pitches, a speaker or singer can choose to phonate using either mechanism M1 
Table I. Counterparts of the laryngeal mechanisms found in the literature, in the case when a vocal register is defined as a laryngeal entity. A question mark underlines a possible uncertainty in the equivalence.

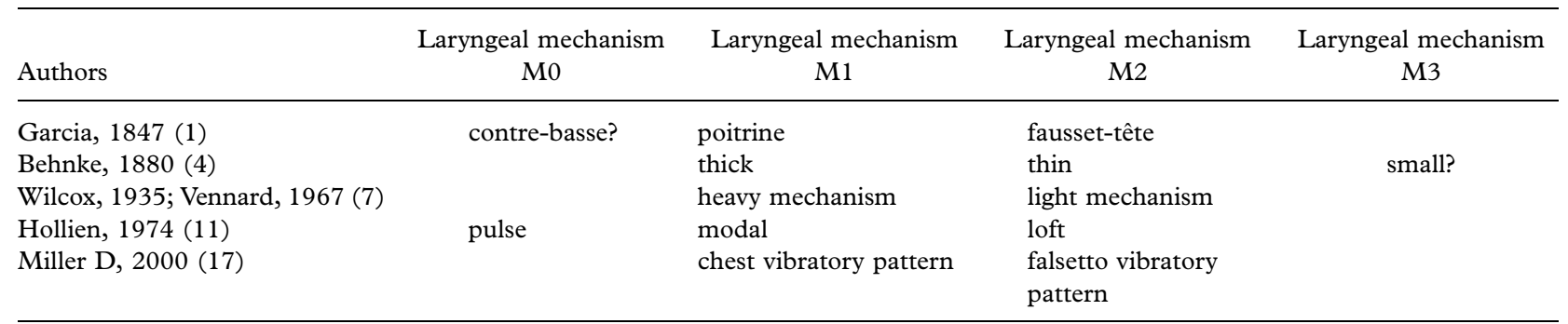

or M2, and this choice may be voluntary or involuntary. In singing, it may be related to the singer's desire to produce a specific voice quality. Indeed, voice quality is dependent on the glottal flow properties. Because the laryngeal mechanisms correspond to different vocal fold vibratory mechanisms, they imply large differences in the glottal flow pulse shape. Their use results in the production of a specific voice quality in relation to the laryngeal configuration. However, skilled singers, and in particular classically trained singers, manage to modify this typical voice quality, in particular in adjusting their vocal tract in the proper way. This is for example the case of voix mixte (mixed voice) register, as will be seen in the next section.

\section{Insights into the physiological, acoustic, and perceptual characteristics of singing voice registers}

In this section, a survey of the most common singing voice registers mentioned in the literature is given. In each case, their main physiological, acoustic, and perceptual characteristics are briefly described. The choice has been made to take the underlying laryngeal mechanism in which they are produced as a common denominator for categorizing these registers. The special case of the middle or voix mixte register is discussed at the end of the section.
Registers produced in laryngeal mechanism M0: pulse, vocal fry or strohbass

Pulse, vocal fry or strohbass are equivalent labels (11). They designate the register which occurs in the lowest part of the human frequency range. This register is produced in laryngeal mechanism M0. The vocal folds are very short, thick and slack. A recent survey of the acoustic, aerodynamic, physiological, and perceptual characteristics of this register in speech can be found in (34). From a physiological point of view, the thyroarytenoid muscles tend to shorten, subsequently thickening the anterior-posterior dimension of the vocal folds, and allowing the anterior part of the vocal ligaments to vibrate (11). The glottal vibratory pattern is characterized by short pulses, which can be periodic and single, periodic and multiple (double, triple), or aperiodic (single and multiple) (34-36). The pulses are followed by a long closed interval $(36,37)$, which implies small values of open quotient. The frequency range goes from a few $\mathrm{Hz}$ to about $80 \mathrm{~Hz}$ with a mean of $50 \mathrm{~Hz}$, and is similar for males and females $(34,38)$. This register is more commonly found in speech than in singing. However, few exceptions can be found in certain vocal cultures around the world (e.g., Russian and Papua New Guinea folk music, rhythm and blues singing style, (15)).

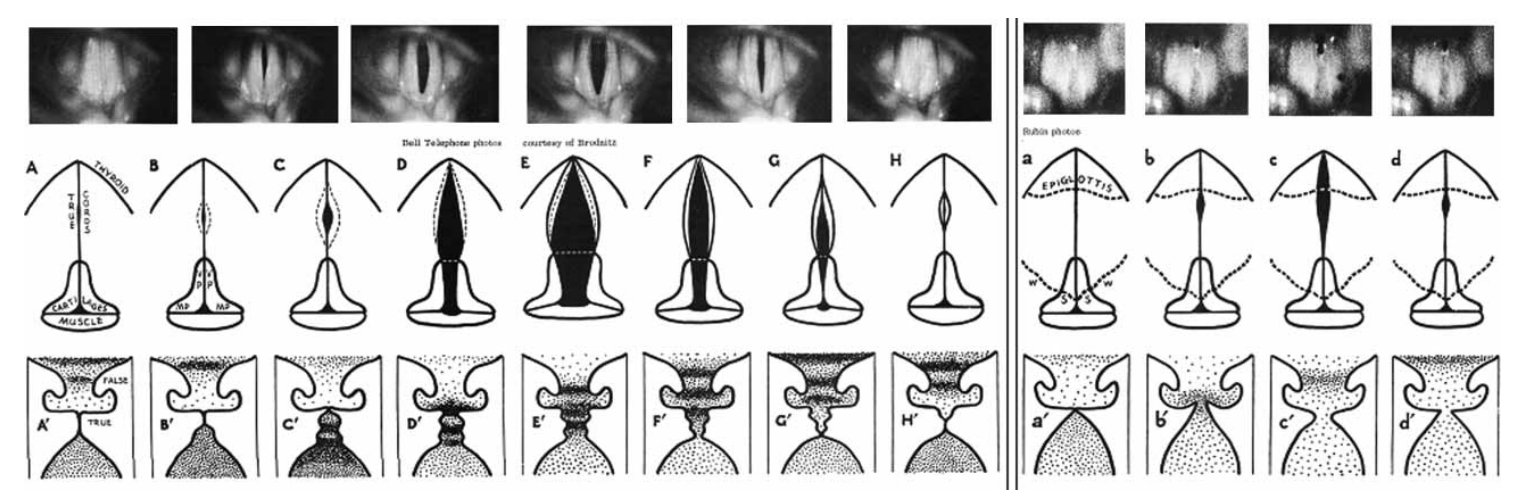

Figure 6. Schematic representation of the vocal folds vibratory movements in laryngeal mechanism M1 and M2. From (7). 


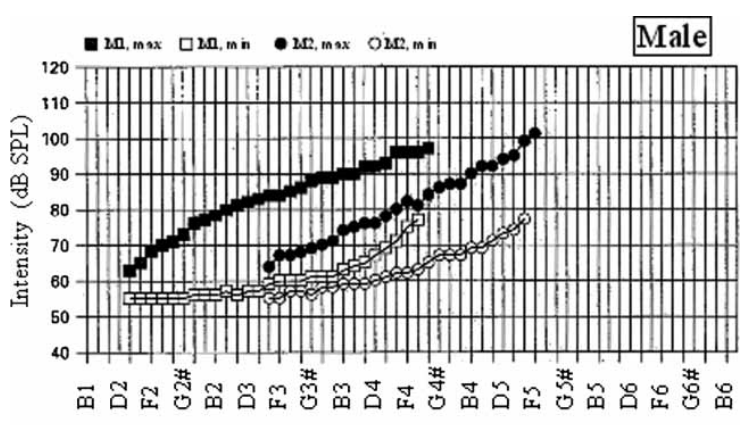

pitches

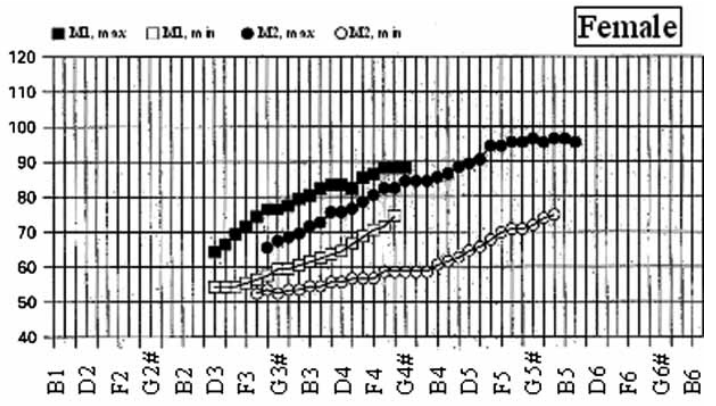

pitches

Figure 7. Average voice range profile (vocal intensity versus fundamental frequency) of male and female singers in mechanisms M1 and M2. Adapted from (33).

Registers produced in laryngeal mechanism M1: modal, chest, male head, belting

The low to mid frequency part of the voice can be divided into a single register (in this case modal or chest register), or into two or more different registers (chest, male head, belting, voix mixte, voce finta,...). The modal, chest, male head, belting, and voce finta registers are produced in laryngeal mechanism M1 $(11,13,39,40)$. The voix mixte register will be described later and can be produced either in $\mathrm{M} 1$ or in M2 (41). The vocal folds are thick, with great vibrating mass and large vibratory amplitude. The inverse-filtered glottal-flow pulse is asymmetrical, the closing phase being shorter than the opening. The open quotient values range from 0.3 to 0.8 , depending on vocal intensity (27).

The distinction between chest and male head registers is defined by Miller (42) in terms of vocal tract adjustment: 'the chest register comprises the lower portion of the range, extending to the highest pitch where the first formant matches the second harmonic; the head register comprises that portion of the range where F1 falls below H2.' The head register is used in the upper range and spans a musical interval of a fourth or fifth (39), located in the vicinity of $\mathrm{D} 4 \mathrm{~b}$ to $\mathrm{A} 4 \mathrm{~b}$ for a tenor and $\mathrm{B} 3 \mathrm{~b}$ to $\mathrm{F} 4$ for a baritone (42). Suitable for soft singing, the head voice is recognized by Vennard as an intermediate adjustment, (...), easily confused with falsetto but continuous with full voice' ((7), paragraph 935). From a laryngeal muscular point of view, head register is characterized by higher cricothyroid activity and lower vocalis activity than chest (26). Yet, it is clearly produced in laryngeal mechanism M1. As pointed out by Large (39), it is 'a different laryngeal mechanism from falsetto', with complete vocal fold approximation, greater energy in the higher partials and lower air flow rate than falsetto.
Belting is very often considered as a technique of loud singing commonly found in non-classical singing, with a voice quality similar to a yell $(40,43-45)$. However, a few authors consider it nowadays as a vocal register on its own (17), and thus it seems that it is worth mentioning here. It could be produced by males and females, but only female voices have been studied up to now. Considered as an extension of chest register into the upper part, its frequency range goes from E4-G4 (about $330 \mathrm{~Hz}$ ) to D5 $(540 \mathrm{~Hz}$ ) (40). Belting sounds are produced with high subglottal pressure, long closed phase of duration greater than half of the fundamental period, high SPL, and great harmonic richness $(40,43,46)$. A tuning of the first formant with the second harmonic has been observed on open vowels $(40,43)$.

Registers produced in laryngeal mechanism M2: falsetto, loft, female head, upper

The falsetto, loft, and female head are equivalent labels for registers which occur in the mid to high frequency part of the voice. The falsetto register can be used to designate altogether a female-like voice quality produced by males, in particular counter tenors, a soft and breathy fake voice quality produced by untrained male singers in the high range, and the female mid to high register, or female head register.

All these registers are produced in laryngeal mechanism M2. The vocal folds are thin and the vibrating mass and vibratory amplitude are reduced. The inverse filtered glottal flow pulse is more symmetrical, close to a sinusoidal shape. The open quotient values are high, ranging from 0.5 to 0.95 depending on the fundamental frequency (27). Their main acoustic characteristic is the predominance of the first harmonic, in relation to high values of the open quotient (47).

In the female case, the mid to high frequency part of the voice may be divided into two or more 
registers, for instance lower middle, upper middle, and upper (48). The boundary between middle and upper registers is located around D5 to F5 (17). According to Miller (17), the main differences between these registers lie in the resonance strategies adopted by the singer. When the fundamental frequency is getting high enough to be close to the first formant, there is a tendency to tune the first formant to the first harmonic, and to maintain this tuning for higher pitches (49-51). The F1-H1 tuning on open vowels would be the determinative feature of the upper register (17).

Registers produced in laryngeal mechanism M3: bell, flute, whistle, flageolet

The bell, flute, whistle, or flageolet are equivalent labels for a register which occurs in the highest part of the human frequency range (52). It is produced in laryngeal mechanism M3. However, the phonatory mechanism has not been explored much. There may be several types of realization (15). Only a few studies have paid attention to this register, which is not much used in speech and classical singing, except in the high soprano range (48). It can be found in non-classical singing styles, such as jazz or contemporary music. The fundamental frequency typically ranges from B5 to F6, i.e., from about 1000 to $1400 \mathrm{~Hz}$ (17). The first harmonic is dominant, and the number of overtones is reduced $(52,53)$. According to Miller, this register is used when the fundamental frequency is too high to remain close to the first formant. The corresponding sound quality is 'small and thin', but nevertheless 'brilliant', comparable to 'a chime or a sharply-ringing distant bell' (48).

What about the registers in the middle of the frequency range: mid, middle, mixed voice, voix mixte?

A special case are the registers found in the middle of the human frequency range, and often labelled as mid, middle, mixed voice, or voix mixte. The term head can also be found (18), and the female middle register can sometimes be considered to be similar to male full head register (17). Yet, the correspondence between these terms cannot be stated with certainty.

Singers make use of these registers to smooth the transition between the lower and upper part of their frequency range, in the frequency region where the frequency ranges of laryngeal mechanisms $M 1$ and M2 overlap. For a long time, it has been queried whether this register would be a mixture of two registers. Recently, it has been demonstrated that, at least from the laryngeal point of view, singers use a precise laryngeal mechanism, which is either M1 or
M2 (41). Male singers very easily use the laryngeal mechanism M1 to produce sounds in voix mixte, whereas female singers very easily use the laryngeal mechanism M2. However, examples have also been studied of a soprano singing in voix mixte register using laryngeal mechanism $\mathrm{M} 1$, or a counter tenor singing in voix mixte register using laryngeal mechanism M2 (41).

The resonance properties of these registers still need to be explored, so as to understand how a singer manages to mimic the voice quality of a given register while using an inappropriate laryngeal mechanism.

\section{Conclusion}

Since Garcia, knowledge about singing voice registers has evolved, thanks to the development of new experimental techniques. Yet, the controversy about vocal registers, which already existed in Garcia's time, is still present nowadays, and reflected in the multiplicity of labels for registers. It seems to have its roots in the definition of a register and its defining characteristics. If a vocal register is defined as a series of consecutive tones produced by the same laryngeal mechanism, the human voice can be characterized by four different voice registers, i.e., laryngeal registers such as Hollien's pulse, modal, loft registers, and the whistle register. If a vocal register is defined as series of consecutive tones produced with similar voice quality, other registers exist in the human singing voice, such as head, belting, middle, upper, voix mixte,... One major issue is then to define a vocal register precisely before labelling it. Another major issue is to be precise about the means which are required to identify a given vocal register. The identification of vocal registers can benefit from the detection of transition phenomena. In this way, the concept of laryngeal mechanism, which is based on the detection of laryngeal transition phenomena by means of electroglottography, is of great help in clarifying the laryngeal nature of a given voice production. At least, the singing voice registers can now be described in terms of the laryngeal mechanism by which they are produced. Further research is needed to understand the singers' resonance strategies and acoustic adaptations which are required to produce a specific voice quality or to successfully manage blending of singing voice registers. Furthermore, major voice quality differences are avoided in the Western lyric culture, whereas they may be a prime goal in other vocal cultures, such as jazz, blues and rock. Knowledge of singing voice registers would gain from a better understanding of these non-classical phonation types. 


\section{Acknowledgements}

The author wants to express her appreciation to Evelyn Abberton for her kind help with the English text.

\section{Notes}

1. English translation of ((1), p. 4) ((54) as mentioned by ((17), p. 30)): 'Par le mot registre, nous entendons une série de sons consécutifs et homogènes allant du grave à l'aigu, produits par le développement du même principe mécanique, et dont la nature diffère essentiellement d'une autre série de sons également consécutifs et homogènes, produits par un autre principe mécanique. Tous les sons appartenant à un même registre sont, par conséquent, de la même nature, quelles que soient d'ailleurs les modifications de timbre ou de force qu'on leur fasse subir.'.

2. 'La séparation [entre registres de fausset et de tête] n'est pas le résultat d'un mécanisme différent du larynx, mais un effet de timbre (...)' ((1), p. 18) - 'The division [between falsetto and head registers] does not result from a different mechanism of the larynx, but from a timbre effect (...)'.

\section{References}

1. Garcia M. Mémoire sur la voix humaine présenté à l'Académie des Sciences en 1840, 2 ed. Paris: Imprimerie d'E. Duverger; 1847.

2. Garcia M. Traité complet de l'Art du Chant, 8 ed. Paris: Heugel et Cie; 1884.

3. Castellengo M. Manuel Garcia Jr, a clear-sighted observer of human voice production. PEVOC 6: London, 2005. Submitted to Logopedics Phoniatrics Vocology.

4. Behnke E. The mechanism of the human voice, 12 ed. London: J. Curwen \& Sons, Warwick Lane, E.C; 1880.

5. Garcia M. Observations on the human voice. In: Proceedings of the Royal Society of London; 1855. London: The Royal Society; p. 399-410.

6. Thurman L, Welch G, Theimer A, Klitzke C. Addressing vocal register discrepancies: an alternative, science-based theory of register phenomena. In: Second International Conference on Physiology and Acoustics of Singing; 6-9 October 2004. Denver, Colorado, USA; 2004. http:// www.ncvs.org/pas/2004/pres/thurman/Thurman.pdf

7. Vennard WL. Singing: the mechanism and the technic. New York: Carl Fischer; 1967.

8. Mörner M, Fransson F, Fant G. Voice register terminology and standard pitch. STL-QPSR. 1963:17-23.

9. Van den Berg JW. Vocal ligaments versus registers. NATS Bulletin. 1963;20:16-21.

10. Hirano M, Vennard W, Ohala J. Regulation of register, pitch and intensity of voice. Folia Phoniatr. 1970;22:1-20.

11. Hollien H. On vocal registers. Journal of Phonetics. 1974;2: $125-43$.

12. Hollien H. Report on vocal registers. In: Askenfelt A, Felicetti S, Jansson E, Sundberg J, editors. Stockholm Musical Acoustic Conference (SMAC); 1983. Stockholm, Sweden: Royal Swedish Academy of Music No. 46:1; 1983. p. $27-35$.

13. Roubeau B. Mécanismes vibratoires laryngés et contrôle neuro-musculaire de la fréquence fondamentale. Université Paris-Orsay; 1993.

14. Roubeau B, Castellengo M. Revision of the notion of voice register. In: XIXth International CoMeT Congress; 1993. Utrecht; 1993.
15. Sakakibara K-I. Production mechanism of voice quality in singing. Journal of the Phonetic Society of Japan. 2003;17(3): $27-39$.

16. Large JW. Towards an integrated physiologic-acoustic theory of vocal registers. NATS Bulletin. 1972;29:18-40.

17. Miller DG. Registers in singing: empirical and systematic studies in the theory of the singing voice. Groningen: University of Groningen; 2000.

18. Titze IR. "Principles of Voice Production. Englewood Cliffs, New Jersey: Prentice Hall; 1994.

19. Titze IR. A framework for the study of vocal registers. J Voice. 1988;2(3):183-94.

20. Castellengo M, Roubeau B, Valette C. Study of the acoustical phenomena characteristic of the transition between chest voice and falsetto. In: Askenfelt A, Felicetti S, Jansson E, Sundberg J, editors. Stockholm Musical Acoustic Conference (SMAC); 1983. Stockholm, Sweden: Royal Swedish Academy of Music No. 46:1; 1983. p. 113-23.

21. Miller DG, Svec JG, Schutte HK. Measurement of characteristic leap interval between chest and falsetto registers. J Voice. 2002;16(1):8-19.

22. Roubeau B, Chevrie-Muller C, Arabia C. Control of laryngeal vibration in register change. In: Gauffin J, Hammarberg B, editors. Vocal Fold Physiology: Acoustic, perceptual, and physiological aspects of voice mechanisms. USA: Singular Publishing Group, San Diego; 1991. p. 279-86.

23. Svec JG, Schutte HK, Miller DG. On pitch jumps between chest and falsetto registers in voice: data from living and excised human larynges. J Acoust Soc Am. 1999;106(3): $1523-31$.

24. Castellengo M. Continuité, rupture, ornementation, ou les bons usages de la transition entre deux modes d'émission vocale. In: Georg, editor. Cahiers de musiques traditionnelles $-\mathrm{La}$ voix et les techniques vocales. Ateliers d'ethnomusicologie, Geneva, Switzerland; 1991.

25. Van den Berg JW, Vennard W, Burger D, Shervanian CC. Voice production: the vibrating larynx. In: Instructional film. University of Groningen ed. The Netherlands; 1960.

26. Hirano M. The role of the layer structure of the vocal fold in register control. Vox Humana. University of Jyvaskyla; 1982. p. $50-62$.

27. Henrich N, d'Alessandro C, Castellengo M, Doval B. Glottal open quotient in singing: Measurements and correlation with laryngeal mechanisms, vocal intensity, and fundamental frequency. J Acoust Soc Am. 2005;117(3):1417-30.

28. Roubeau B, Chevrie-Muller C, Arabia-Guidet C. Electroglottographic study of the changes of voice registers. Folia Phoniatr. 1987;39(6):280-9.

29. Henrich N, Roubeau B, Castellengo M. On the use of electroglottography for characterisation of the laryngeal mechanisms. In: Stockholm Music Acoustics Conference; 2003. Stockholm, Sweden; 2003. http://www.speech.feth.se/ $\operatorname{smac03/}$

30. Lecluse F, Brocaar M. Quantitative measurements in the electroglottogram. In: 17th International Congress of Logopedics and Phoniatrics. 1977.

31. Hirano $M$. Vocal mechanisms in singing: laryngological and phoniatric aspects. J Voice. 1988;2(1):51-69.

32. Herzel H, Reuter R. Whistle register and biphonation in a child's voice. Folia Phoniatr Logop. 1997;49:216-24.

33. Roubeau B, Castellengo M, Bodin P, Ragot M. Phonétogramme par mécanisme laryngé. [Laryngeal registers as shown in the voice range profile]. Folia Phoniatr Logop. 2004;56(5):321-33.

34. Blomgren $\mathrm{M}$, Chen $\mathrm{Y}, \mathrm{Ng} \mathrm{ML}$, Gilbert HR. Acoustic, aerodynamic, physiologic, and perceptual properties of modal 


\section{N. Henrich}

and vocal fry registers. J Acoust Soc Am. 1998;103(5):2649 58.

35. Childers DG, Lee CK. Vocal quality factors: Analysis, synthesis, and perception. J Acoust Soc Am. 1991;90:2394410.

36. Whitehead RL, Metz DE, Whitehead BH. Vibratory patterns of the vocal folds during pulse register phonation. J Acoust Soc Am. 1984;75(4):1293-7.

37. Hollien H, Girard GT, Coleman RF. Vocal fold vibratory patterns of pulse register phonation. Folia Phoniatr. 1977;29: 200-5.

38. Hollien H, Michel JF. Vocal fry as a phonational register. J Speech Hear Res. 1968;11:600-4.

39. Large JW. Male high voice mechanisms in singing. Journal of Research in Singing. 1984;VIII(1):1-10.

40. Schutte HK, Miller DG. Belting and pop, nonclassical approaches to the female middle voice: some preliminary considerations. J Voice. 1993;7(2):142-50.

41. Castellengo $\mathrm{M}$, Chuberre $\mathrm{B}$, Henrich $\mathrm{N}$. Is voix mixte, the vocal technique used to smooth the transition across the two main laryngeal mechanisms, an independent mechanism? In: ISMA; 2004. Nara, Japan; 2004.

42. Miller DG, Schutte HK. Toward a definition of male 'head' register, passaggio, and 'cover' in western operatic singing. Folia Phoniatr Logop. 1994;46(4):157-70.

43. Bestebreurtje ME, Schutte HK. Resonance strategies for the belting style: results of a single female subject study. J Voice. 2000;14(2):194-204.

44. Estill J. Belting and classic voice quality: some physiological differences. Medical Problems of Performing Artists. 1988:37-43.
45. Miles B, Hollien H. Whither belting? J Voice. 1990;4(1):6470 .

46. Sundberg J, Gramming P, Lovetri J. Comparisons of pharynx, source, formant, and pressure characteristics in operatic and musical theatre singing. J Voice. 1993;7(4):301-10.

47. Sundberg J, Hogset C. Voice source differences between falsetto and modal registers in counter tenors, tenors and baritones. Logoped Phoniatr Vocol. 2001;26(1):26-36.

48. Miller R. English, French, German and Italian techniques of singing: a study in national tonal preferences and how they relate to functional efficiency. Metuchen, N.J.: The Scarecrow Press, Inc; 1977.

49. Joliveau E, Smith J, Wolfe J. Vocal tract resonances in singing: the soprano voice. J Acoust Soc Am. 2004;116(4 Pt1):24349.

50. Sundberg J. Observations on a professional soprano singer. STL-QPSR. 1973;14(1):14-24.

51. Sundberg J. Formant technique in a professional soprano singer. Acustica. 1975;32:89-96.

52. Miller DG, Schutte HK. Physical definition of the flageolet register. J Voice. 1993;7(3):206-12.

53. Walker JS. An investigation of whistle register in the female voice. J Voice. 1988;2:140-50.

54. Paschke DVA. Complete Treatise on the Art of Singing: Part One (Translation of Garcia, 1847). New York: Da Capo Press; 1984.

55. Henrich N, d'Alessandro C, Castellengo M, Doval B. On the use of the derivative of electroglottographic signals for characterization of nonpathological phonation. J Acoust Soc Am. 2004;115(3):1321-32. 